https://helda.helsinki.fi

Preliminary case control study to establish the correlation between novel peroxidation biomarkers in cord serum and the severity of hypoxic ischemic encephalopathy

\title{
Chafer, Consuelo
}

2016

Chafer , C , Cernada , M , Rahkonen , L, Andersson, S , Stefanovic , V \& Vento , M 2016 , ' Preliminary case control study to establish the correlation between novel peroxidation biomarkers in cord serum and the severity of hypoxic ischemic encephalopathy ', Free Radical Biology \& Medicine , vol. 97 , pp. 244-249 . https://doi.org/10.1016/j.freeradbiomed.2016.06.006

http://hdl.handle.net/10138/224016

https://doi.org/10.1016/j.freeradbiomed.2016.06.006

publishedVersion

Downloaded from Helda, University of Helsinki institutional repository.

This is an electronic reprint of the original article.

This reprint may differ from the original in pagination and typographic detail.

Please cite the original version. 
Original article

\title{
Preliminary case control study to establish the correlation between novel peroxidation biomarkers in cord serum and the severity of hypoxic ischemic encephalopathy
}

\author{
Sture Andersson ${ }^{\mathrm{d}}$, Máximo Vento ${ }^{\mathrm{a}, \mathrm{b}, *}$ \\ ${ }^{a}$ Neonatal Research Group, Health Research Institute La Fe, Valencia, Spain \\ ${ }^{\mathrm{b}}$ Division of Neonatology, University \& Polytechnic Hospital La Fe, Valencia, Spain \\ ${ }^{\mathrm{c}}$ Department of Obstetrics and Gynecology, Fetomaternal Medical Center, Helsinki University Hospital, Finland \\ ${ }^{\mathrm{d}}$ Children's Hospital, University of Helsinki, and Helsinki University Hospital, Helsinki, Finland
}

Consuelo Chafer-Pericas ${ }^{a}$, María Cernada ${ }^{a}$, Leena Rahkonen ${ }^{c}$, Vedran Stefanovic ${ }^{c}$,

\section{A R T I C L E I N F O}

\section{Article history:}

Received 8 March 2016

Received in revised form

1 June 2016

Accepted 9 June 2016

Available online 11 June 2016

Keywords:

Birth asphyxia

Biomarker

Delivery

Hypoxia

Isoprostanes

Lactic acid

Metabolic acidosis

Oxidative stress

\begin{abstract}
A B S T R A C T
Background: Hypoxic-ischemic encephalopathy (HIE) has deleterious neurological consequences. To identify patients at risk of neuronal damage deserving implementation of neuroprotective strategies clinicians have relied on prenatal sentinel events, postnatal clinical assessment (Apgar score), and blood gas analysis.

This feasibility study aimed to assess if lipid peroxidation byproducts associated with neuronal damage correlated with cord blood metabolic acidemia in patients with HIE.

Population and Methods: This is a case/control study in which cases were newborn infants with severe acidemia $(\mathrm{pH}<7.00$; base excess $\geq 12 \mathrm{mmol} / \mathrm{L})$ while control babies exhibited normal gases $(\mathrm{pH}=7.20$ 7.40; base excess $=-4$ to $+4 \mathrm{mmol} / \mathrm{L}$ ) in the first cord blood analysis performed immediately after birth. Concomitantly, lipid peroxidation byproducts were determined using ultra performance liquid chromatography coupled to mass spectrometry in the same cord blood sample.

Results: A total of 19 controls and 20 cases were recruited. No differences in gestational characteristics were present. However, cases exhibited profound metabolic alterations as compared to controls (Cases vs. Control: $\mathrm{pH}=6.90 \pm 0.1$ vs. $7.33 \pm 0.03$; base excess $=-15 \pm 3$ vs. $-1 \pm 2 \mathrm{mmol} / \mathrm{L}$ ), $85 \%$ were admitted to the NICU, and 50\% developed symptoms of HIE. 8-iso-15(R)-PGF2 $\alpha(\mathrm{P}=0.01)$ and total isoprostanes $(\mathrm{P}=0.045)$ presented statistically significant differences between cases and control groups and correlated with level of HIE.

Conclusions: The 8-iso-15(R)-PGF2 $\alpha$ and isoprostanes reflecting oxidative damage are significantly increased in severe postnatal acidemia. Follow up studies with adequate power are necessary to confirm if these biomarkers measured in cord blood serum could be predictive of neonatal encephalopathy.
\end{abstract}

(c) 2016 Elsevier Inc. All rights reserved.

\section{Introduction}

Impaired gas exchange during labor leads to hypoxemia, hypercapnia, and metabolic acidosis that if severe enough may cause neonatal encephalopathy [1]. Brain cells are highly dependent on aerobic metabolism to obtain energy for ATP powered ion pumps, synaptic activity, and redox status. Intense hypoxia and re-oxygenation with high oxygen concentrations may lead to cell swelling, hyperexcitability and oxidative stress [2]. Altogether these

\footnotetext{
* Correspondening author at: Neonatal Research Group, Health Research Institute La Fe, University and Polytechnic Hospital La Fe, Avenida Fernando Abril Martorell 106, 46026 Valencia, Spain.

E-mail address: maximo.vento@uv.es (M. Vento).
}

circumstances may cause neuronal necrosis or activate pro-apoptotic pathways that lead to amplification of the initial damage evolving for hours, days or even weeks [3]. Injury can occur as intracranial hemorrhage or infarction, or the more global injury of hypoxic-ischemic encephalopathy (HIE). HIE occurs in 1-8 per 1000 live births in developed countries. Notably, around 30\% of the affected newborns will die in the postnatal period, and a significant number of survivors will develop severe and permanent neurocognitive, motor or sensorial sequel [4].

In perinatal asphyxia, blood gas analysis of arterial cord samples is characterized by a profound metabolic acidemia with increased lactate concentration. However, lactate production can be induced by non-hypoxic conditions such as glycogenolysis, alkalosis, or catecholamine infusion. Therefore, the reliability of the analysis of cord 
blood gases is of limited value in predicting HIE [5-7]. Moreover, the clinical methods employed to assess HIE which include Apgar score, amplitude integrated electroencephalography, and magnetic resonance imaging are useful for predicting some outcome of neonatal hypoxia but lack the sensitivity needed to implement effective therapies such as hypothermia [8].

For these reasons, there has been a protracted search for biomarkers assessing brain damage and predicting neurocognitive outcome in the neonatal population. The aim of these markers would be to early detect and monitor neuronal damage, to study the pathogenesis of brain damaging disorders, and finally being reliable in predicting outcome [9]. To date the most widely employed encompass among others S100B, neuron-specific enolase, activin $A$, adrenomedullin, Interleukin $1 \beta$, and IL6. However, increased levels in different biofluids of these biomarkers do not always correspond to brain damage. Moreover, these metabolites are not brain specific and may be released by tissues different than brain [10]. Recently, highly specific proteins such as glial fibrillary acidic protein (GFAP) and ubiquitin carboxy-terminal hydrolase L1 (UCH-L1), markers of gliosis and neuronal injury, respectively, have been employed to assess neuronal damage in HIE. Notably, while these biomarkers have shown to be useful in adult brain conditions, findings in the neonatal patients have been somewhat limited. Although some studies shed promising results for both biomarkers for assessment of the degree of brain damage and later neurodevelopmental outcomes [11], more recently it has been confirmed that there is no correlation between cord blood samples and HIE staging and later neurodevelopmental outcomes. Thus, GFAP and UCH-L1 plasma levels correlate with the severity of HIE and with neurocognitive outcome when determinations are made between 6 and $12 \mathrm{~h}$ after birth [12,13].

The role of oxidative stress biomarkers has been reviewed [14]. In the experimental setting metabolomic studies performed in animal models of hypoxia reoxygenation have shown that oxidative stress associated metabolites strongly correlate with brain injury. The most specific and reliable biomarkers identified have been isoprostanes, neuroprostanes, non-protein bound iron, and 4-HNE proteins adducts [15-17]. F2-isoprostanes have been determined to be both biomarkers and mediators of oxidative stress in numerous diseases [18]. Actually, a previous study revealed that F2-isoprostanes increased after ischemia-reperfusion injury in humans [19]. In a rat model of global perinatal asphyxia, increased brain levels of F2-isoprostanes were found in neonatal rats after 20-min asphyxia, as well as alterations in behavioral patterns [20]. Also, high 8-isoprostane levels were found in cerebrospinal fluid from preterm infants with evidence of white matter injury on magnetic resonance imaging at term [21].

The aim of this study was to identify if markers of lipid peroxidation validated in the newborn period significantly correlated with severe metabolic acidosis determined in cord blood serum immediately after birth using ultra performance liquid chromatography-mass spectrometry methodology.

\section{Material and methods}

\subsection{Study design and participants}

This is a prospective case-control observational study performed at the Division of Obstetrics and Gynecology of the Helsinki University Central Hospital (HUCH) during a 12-month period. The Ethics Committee of the HUH approved the study protocol and informed consent was obtained from the parents of all recruited newborn infants.

Eligible participants were inborn newly born infants with gestational age between 37 and 42 weeks. Patients were classified in two groups according to the umbilical cord arterial blood $\mathrm{pH}$, base excess and $\mathrm{pCO}_{2}$ obtained immediately after birth: control group $\left(\mathrm{pH}=7.20\right.$ to 7.40 , base excess $=4$ to $\left.-4 \mathrm{mEq} / \mathrm{L}, \mathrm{pCO}_{2}=5-6.9 \mathrm{kPa}\right)$ and case group $\left(\mathrm{pH} \leq 7.05\right.$, base excess $=\leq 12 \mathrm{mEq} / \mathrm{L}, \mathrm{pCO}_{2}$ $>8 \mathrm{kPa})$.

\subsection{Sample collection and storage}

Serum samples were obtained from cord umbilical artery immediately after cord clamping and before resuscitation maneuvers were initiated. Collected blood was immediately put into a dry cryotube. The tube was held in vertical position and at room temperature to improve the coagulation during $30 \mathrm{~min}$ after the extraction. Then, the sample was centrifuged at $2000 \times g$ for $10 \mathrm{~min}$ at room temperature, and the supernatant (serum) was separated and aliquoted. Thereafter, samples were stored at $-80^{\circ} \mathrm{C}$ until analysis.

\subsection{Reagents}

Isoprostanes' standards of 8-iso-15(R)-PGF ${ }_{2 \alpha}, 1 \mathrm{a}, 1 \mathrm{~b}-$ dihomo$\mathrm{PGF}_{2 \alpha}$, 2,3-dinor-iPF $2 \alpha$-III, 8-iso-15-keto-PGE, 8-iso-15-keto$\mathrm{PGF}_{2 \alpha}$, 8-iso-PGE $2,5-\mathrm{PFF}_{2 \alpha}-\mathrm{VI}, 8$-iso-PGF $2 \alpha$, the prostaglandins $\mathrm{PGE}_{2}$ and $\mathrm{PGF}_{2 \alpha}$, as well as the deuterated internal standard (IS) $\mathrm{PGF}_{2 \alpha}-\mathrm{D}_{4}$ were purchased from Cayman Chemical Company (Ann Arbor, Michigan, USA). Potassium hydroxide was from Sigma Aldrich Química SA (Madrid, Spain). Methanol (LC-MS grade) and n-heptane (analytical grade) were obtained from J.T. Baker (Avantor Performance Materials B.V., The Netherlands). Formic acid (98\%) and ethyl acetate (analytical grade) were from Panreac (Barcelona, Spain).

\subsection{Materials}

SPE-96 well plates (Discovery ${ }^{\circledR}$ DSC-18, $100 \mathrm{mg}$ ) were used for sample solid-phase extraction (SPE) (Sigma-Aldrich, St. Louis, MO, USA). Ultrasonic bath (BandelinSonorexDigitec, Berlin, Germany) was used to improve the hydrolysis efficiency. Vortex mixer was from VelpScientifica (Usmate, Italy). Centrifuge Biocen22R was from OrtoAlresa (Madrid, Spain). Thermomixer HLC from Ditabis (Pforzheim, Germany). Speed vacuum concentrator (mi Vac) was from Genevac LTD (Ipswich, United Kingdom). 96-well sample plates (Acquity UPLC $700 \mu \mathrm{L}$ ) from Waters (Barcelona, Spain).

\subsection{Ultra high performance liquid chromatography-mass spectro- metry chromatographic system (UPLC-MS/MS)}

The chromatographic system used consisted of a Waters Acquity UPLC-Xevo TQD system (Milford, MA, USA). The conditions employed were established in a recently published work [30]. Briefly, negative electrospray ionization $\left(\mathrm{ESI}^{-}\right)$, capillary voltage $3.5 \mathrm{kV}$, source temperature $120^{\circ} \mathrm{C}$, desolvation temperature $300{ }^{\circ} \mathrm{C}$, nitrogen cone and desolvation gas flows were 25 and $680 \mathrm{Lh}^{-1}$, respectively, and dwell time was $5 \mathrm{~ms}$. An Acquity UPLC BEH C18 column $(2.1 \times 50 \mathrm{~mm}, 1.7 \mu \mathrm{m})$ and pre-column $(2.1 \times 5 \mathrm{~mm})$ from Waters, and binary mobile phase $\mathrm{CH}_{3} \mathrm{OH}(0.1 \%$ $\mathrm{v} / \mathrm{v} \mathrm{HCOOH}): \mathrm{H}_{2} \mathrm{O}(0.1 \% \mathrm{v} / \mathrm{v} \mathrm{HCOOH})$ with gradient elution were employed. The flow rate was $0.4 \mathrm{~mL} \mathrm{~min}^{-1}$, the column temperature was $37^{\circ} \mathrm{C}$ and the injection volume was $10 \mu \mathrm{L}$. Mass spectrometric detection was carried out by multiple reaction monitoring (MRM).

\subsection{Sample treatment}

The sample treatment was optimized and assayed as described before [30]. Briefly, serum samples were thawed on ice, and $100 \mu \mathrm{L}$ 
of sample was added to $3 \mu \mathrm{L}$ of internal standard (IS) solution $\mathrm{PGF}_{2 \alpha}$-D4 $20 \mu \mathrm{mol} \mathrm{L}^{-1}$ and to $100 \mu \mathrm{L}$ of potassium hydroxide (15\%, $\mathrm{w} / \mathrm{v}$ ) incubating at $40{ }^{\circ} \mathrm{C}$ for $30 \mathrm{~min}$. Thereafter, the hydrolyzates were diluted with $680 \mu \mathrm{L}$ of $\mathrm{H}_{2} \mathrm{O}(0.1 \% \mathrm{HCOOH}, \mathrm{pH} 3): \mathrm{CH}_{3} \mathrm{OH}$ (85:15 v/v), acidified with $20 \mu \mathrm{L}$ of formic acid (98\%), and homogenized. After that, they were kept in an ice bath for $10 \mathrm{~min}$. Then, they were centrifuged at $4000 \times \mathrm{g}$, at $4{ }^{\circ} \mathrm{C}$ for $10 \mathrm{~min}$. A solid phase extraction (SPE) procedure was carried for clean-up and pre-concentration. Finally, the extracts were injected in the chromatographic system (UPLC-MS/MS).

\subsection{Statistical analysis}

Taking into account the clinical variables most employed in the acidosis diagnosis, samples were classified into two groups, control $(n=19)$ and case $(n=20)$, corresponding to negative and positive diagnosis, respectively.

Univariate statistical analyses were performed using SPSS version 20.0 software (SPSS, Inc., Chicago, IL, USA). For all analyses, the statistical significance was set at $\mathrm{P}<0.05$. Data were presented as mean \pm standard deviation (SD), median (inter-quartile range, IQR) or number of cases (n) and percentage (\%).

Kolmogorov-Smirnov test was used to test for normal distribution of the data. Some parametric demographic and clinical variables were compared between control and case groups using a Student"s $t$-test. Other variables were compared by nonparametric methods as they were not normally distributed. In fact, MannWhitney $U$ tests were performed to assess continuous clinical variables and biomarker concentration differences between groups. Chi square test was employed to compare categorical variables expressed as numbers (percentage, $n$ ).

Receiver operating characteristic (ROC) curve construction was used for diagnostic test evaluation of each biomarker. We calculated the area under the curve (AUC, 95\% confidence interval (CI)), which indicates the global accuracy of a biomarker or a group of biomarkers. The cut-off values in the prediction of metabolic acidosis were established as the highest sum of sensitivity and specificity for each marker in the ROC curve. Diagnostic indices were calculated (sensitivity, specificity, positive predictive value (PPV), negative predictive value (NPV), positive likelihood ratio $(\mathrm{LR}+)$, negative likelihood ratio ( $\mathrm{LR}-$ ) and diagnostic odds ratio (DOR) for the biomarkers to diagnose acidosis.

\section{Results}

\subsection{Patient characteristics}

A total of 39 newborns were enrolled in the study. According to the blood gas analysis obtained in arterial cord blood, consecutive newly born infants were assigned to the control group $(n=19)$ or case group $(n=20)$ as described in Section 2. Patients' demographic and clinical characteristics are shown in Table 1. There were no statistical differences for gestational age at delivery, gender, mode of delivery, birth weight and body mass index, between the control and case groups. However, case group patients exhibited a significantly lower cord $\mathrm{pH}$, base excess, and Apgar scores as compared to patients from control group $(\mathrm{P}<0.001)$. Also, statistical significant differences were obtained for $\mathrm{pCO}_{2}$ between both groups $(\mathrm{P}<0.001)$.

\subsection{Perinatal clinical outcomes}

From the 20 babies that were classified as cases (umbilical artery $\mathrm{pH}<7.05), 17$ were classified as having asphyxia $(85 \%)$. However, 6 of them (30\%) were considered mild and were not
Table 1

Characteristics of studied population.

\begin{tabular}{|c|c|c|c|}
\hline & Case $(n=20)$ & Control $(n=19)$ & P value \\
\hline Maternal age (years), mean \pm SD & $30 \pm 5$ & $33 \pm 6$ & 0.19 \\
\hline Gravida $(\mathrm{n})$, mean \pm SD & $1.7 \pm 0.9$ & $2.7 \pm 1.4$ & 0.01 \\
\hline Parity $(n)$, mean $\pm S D$ & $0.5 \pm 0.7$ & $1.1 \pm 1.0$ & 0.06 \\
\hline $\begin{array}{l}\text { Gestational age at delivery (weeks) } \\
\text { mean } \pm \text { SD }\end{array}$ & $40.3 \pm 1.3$ & $40.6 \pm 1.2$ & 0.5 \\
\hline Mode of delivery global & $12 / 8$ & $9 / 10$ & 0.6 \\
\hline Vaginal total, (\%) & $12(60)$ & $9(47.3)$ & \\
\hline Vaginal + vacuum, (\%) & $5(25)$ & $3(15.8)$ & \\
\hline C-section, (\%) & $8(60)$ & $10(52.6)$ & \\
\hline Birth weight $(\mathrm{g})$, mean \pm SD & $3400 \pm 600$ & $3600 \pm 500$ & 0.4 \\
\hline Umbilical artery $\mathrm{pH}$, mean \pm SD & $6.90 \pm 0.1$ & $7.33 \pm 0.03$ & $<0.001$ \\
\hline $\begin{array}{l}\text { Umbilical artery base excess (mmol/ } \\
\text { L), mean } \pm \text { SD }\end{array}$ & $-15.0 \pm 3$ & $-1.0 \pm 2$ & $<0.001$ \\
\hline $\begin{array}{l}\text { Umbilical artery } \mathrm{pCO}_{2}(\mathrm{kPa}) \\
\text { mean } \pm \mathrm{SD}\end{array}$ & $13 \pm 3$ & $6.4 \pm 0.4$ & $<0.001$ \\
\hline $\begin{array}{l}\text { Umbilical artery } \mathrm{pO}_{2}(\mathrm{kPa}) \\
\text { mean } \pm \mathrm{SD}\end{array}$ & $2.0 \pm 0.9$ & $2.5 \pm 0.8$ & 0.05 \\
\hline Apgar score at $1 \mathrm{~min}$, median (IQR) & $3(1-4)$ & $9(9-9)$ & $<0.001$ \\
\hline Apgar score at 5 min, median (IQR) & $7(6-8)$ & $9(9-9)$ & $<0.001$ \\
\hline Apgar score at $10 \mathrm{~min}$, median (IQR) & $9(6-9)$ & $9(9-9)$ & 0.6 \\
\hline Body mass index, mean \pm SD & $23 \pm 3$ & $25 \pm 7$ & 0.4 \\
\hline
\end{tabular}

Data were expressed as mean $+\mathrm{SD}$ (standard deviation) for parametric continuous variables, number of cases (percentages) for categorical variables or median (interquartile range, IQR) for non-parametric continuous variables. The statistical calculations to compare between the two groups employed Student"s $t$-test, Chi square test or Mann Whitney $U$ test, respectively.

admitted in the NICU. The remaining 11 (55.0\%) were admitted in the NICU (Median length of stay: 2 days (range 1-6)), and $4(20.0 \%)$ were staged as moderate to severe HIE according to clinical criteria from the Helsinki University Children"s Hospital, with initial abnormal structured neurological examination, clinical seizures and abnormal multichannel EEG. Two of these babies received hypothermia treatment. No patient died.

\subsection{Perinatal asphyxia biomarkers}

The compounds detected in cord serum samples were PGE2, 8-isoPGE2, 8-iso-15(R)-PGF2 $\alpha$, 1a,1b-dihomo-PGF2 $\alpha$, 2,3-dinoriPF2 $\alpha$-III, 8 -iso-15-keto-PGF2 $\alpha, 5$-iPF2 $\alpha$-VI, 8-iso-PGF2 $\alpha$, PGF2 $\alpha$, and total neuroprostanes, neurofurans, isoprostanes and isofurans. Results for these metabolites are summarized in Table 2. Comparison of biomarkers using the Mann Whitney $U$ test revealed that the median for 8-iso-15(R)-PGF2 $\alpha(\mathrm{P}=0.01)$ and isoprostanes $(\mathrm{P}=0.045)$ were significantly higher in the case group (Table 2$)$. However, no statistically significant differences were obtained for the rest of the compounds. Box plots for 8-iso-15(R)-PGF2 $\alpha$ and isoprostanes in case and control groups are depicted in Fig. 1.

In addition, we performed ROC curves for 8 -iso-15(R)-PGF2 $\alpha$ and isoprostanes to estimate their diagnostic power (Fig. 2). For 8-iso-15(R)-PGF2 $\alpha$ the AUC was $0.726(\mathrm{P}=0.016)$, with an optimal cut-off value of $4.39 \mathrm{nmol} \mathrm{L}^{-1}$. For isoprostanes the AUC was 0.684 $(\mathrm{P}=0.049)$, with an optimal cut-off value of 0.84 intensity of signal units $\times$ (internal standard concentration, $\left.\mathrm{nmol} \mathrm{L}^{-1}\right)$. 8iso-15(R)PGF2 $\alpha$ and isoprostanes provided good diagnostic accuracy. As shown in Table 3, the LR+ values for 8 -iso-15(R)-PGF2 $\alpha$ reveal that it is good diagnostic biomarker. However, isoprostanes show a smaller increase in the likelihood of having positive test in the case group respect to the control group. The DOR values described the strength of the association between the analysis results and the acidosis occurrence. Since all these values were higher than 1 , this association was statistically significant, being 8-iso-15(R)-PGF2 $\alpha$ the best diagnostic biomarker. 
Table 2

Cord serum isoprostanes' concentrations determined by UPLC-MS/MS for control and case (acidemic) newborn infants.

\begin{tabular}{|c|c|c|c|c|}
\hline Compounds & Case $(n=20)$ & Control $(n=19)$ & P value & $<\operatorname{LOD}(\%)^{\mathrm{a}}$ \\
\hline PGE2 $\left(\mathrm{nmol} \mathrm{L}^{-1}\right)$ & $12(6-76)$ & $25(64-0)$ & 0.88 & 26 \\
\hline 8-isoPGE2 $\left(\mathrm{nmol} \mathrm{L}^{-1}\right)$ & $5(0-13)$ & $0(0-7)$ & 0.18 & 43 \\
\hline $\begin{array}{l}\text { 8-iso-15(R)-PGF2 } \alpha \\
\quad\left(\mathrm{nmol} \mathrm{L}^{-1}\right)\end{array}$ & $2(0-6)$ & $0(0-1.5)$ & $0.01^{*}$ & 46 \\
\hline $\begin{array}{l}\text { 1a,1b-dihomo-PGF2 } \alpha \\
\left(\mathrm{nmol} \mathrm{L}^{-1}\right)\end{array}$ & $0(0-3.4)$ & $0(0-4)$ & 0.95 & 69 \\
\hline $\begin{array}{l}\text { 2,3-dinor-iPF2 } \alpha \text {-III } \\
\quad\left(\mathrm{nmol} \mathrm{L}^{-1}\right)\end{array}$ & $0.0(0.0-0.8)$ & $0.0(0-0.5)$ & 0.56 & 56 \\
\hline $\begin{array}{l}\text { 8-iso-15-keto-PGF2 } \alpha \\
\quad\left(\mathrm{nmol} \mathrm{L}^{-1}\right)\end{array}$ & $3(0-9)$ & $1(0-4)$ & 0.24 & 33 \\
\hline 5-iPF2 $\alpha-\mathrm{VI}\left(\mathrm{nmol} \mathrm{L}^{-1}\right)$ & $3(1-13)$ & $1.7(1.0-3.7)$ & 0.15 & 15 \\
\hline 8 -iso-PGF2 $\alpha\left(\mathrm{nmol} \mathrm{L}^{-1}\right)$ & $0.0(0.0-0.3)$ & $0(0-0)$ & 0.12 & 74 \\
\hline PGF $2 \alpha\left(\mathrm{nmol} \mathrm{L}^{-1}\right)$ & $5(3-10)$ & $4(3-6)$ & 0.13 & 0 \\
\hline Neuroprostanes ${ }^{\mathrm{b}}$ & $0.0(0-0.4)$ & $0.0(0-0.4)$ & 0.95 & 51 \\
\hline Neurofurans ${ }^{\mathrm{b}}$ & $1(0-2)$ & $0.3(0-1.0)$ & 0.08 & 23 \\
\hline Isoprostanes ${ }^{\mathrm{b}}$ & $0.9(0.1-4.4)$ & $0.4(0-0.8)$ & $0.045^{*}$ & 33 \\
\hline Isofurans ${ }^{\mathrm{b}}$ & $1.3(0.7-20)$ & $0.7(0.0-3.1)$ & 0.11 & 23 \\
\hline
\end{tabular}

Values are given as median (inter-quartile range). Mann Whitney $U$ test was used for comparison. Median calculated considering zero the values lower than the limit of detection (LOD).

* $\mathrm{P}<0.05$.

a \% Samples with concentrations of the corresponding analyte < LOD.

b Total parameters results are expressed as (intensity of signal units $\times$ (internal standard concentration, $\left.\mathrm{nmol} \mathrm{L}^{-1}\right)$ ).

\subsection{Correlation between perinatal asphyxia degree and PGF Iso- prostane in cord serum}

The concentration of 8-iso-15(R)-PGF2 $\alpha$ in cord serum correlated significantly $(\mathrm{p}<0.05)$ with the degree of asphyxia. Hence, mean serum concentration of 8-iso-15(R)-PGF2 $\alpha$ was $2.02 \mathrm{nmol} / \mathrm{L}$, $4.98 \mathrm{nmol} / \mathrm{L}$ and $6.43 \mathrm{nmoL} / \mathrm{L}$ in non-asphyctic, mildly asphyctic, and moderate to severely asphyctic respectively.

\section{Discussion}

In a prospective single center study completed in Belgium, cord blood was analyzed for every newborn for a two-year period totalizing of 6614 newborn infants. Metabolic acidosis defined as an arterial cord $\mathrm{pH} \leq 7.05$ with or without venous $\mathrm{pH} \leq 7.17$ in conjunction with base excess of $\leq 10 \mathrm{mmol} / \mathrm{L}$ was present in $0.9 \%$ of deliveries, and in $0.1 \%$ there were transient neurologic sequel but unluckily no long term follow up was performed [23]. Other studies have tried to identify alterations of umbilical artery blood $\mathrm{pH}$, base excess or even lactate with term infants at risk of morbidity [6,24-26]. Notably, despite all these efforts the correlation between low umbilical artery $\mathrm{pH}$, base excess or even lactate and neurological outcome has not been clearly established and most term neonates with low $\mathrm{pH}$, elevated base excess, or even elevated lactate develop normally $[27,28]$. Given the possibility of neuroprotection for babies at risk of HIE $[3,4]$, it would be desirable to dispose of early and robust biomarkers capable of predicting outcomes and helping neonatologist to establish therapeutic indication.

The aim of this work was to explore the correlation of oxidative stress biomarkers with the clinical variables employed for metabolic acidosis diagnosis, and allow establishing a diagnosis from clinically undefined samples.

Brain is strikingly dependent on aerobic metabolism to satisfy its elevated energy requirements and therefore, highly susceptible even to short periods of hypoxia [2]. Lack of oxygen causes exhaustion of ATP and accumulation of purine derivatives, especially hypoxanthine. Upon re-oxygenation, xanthine oxidase using
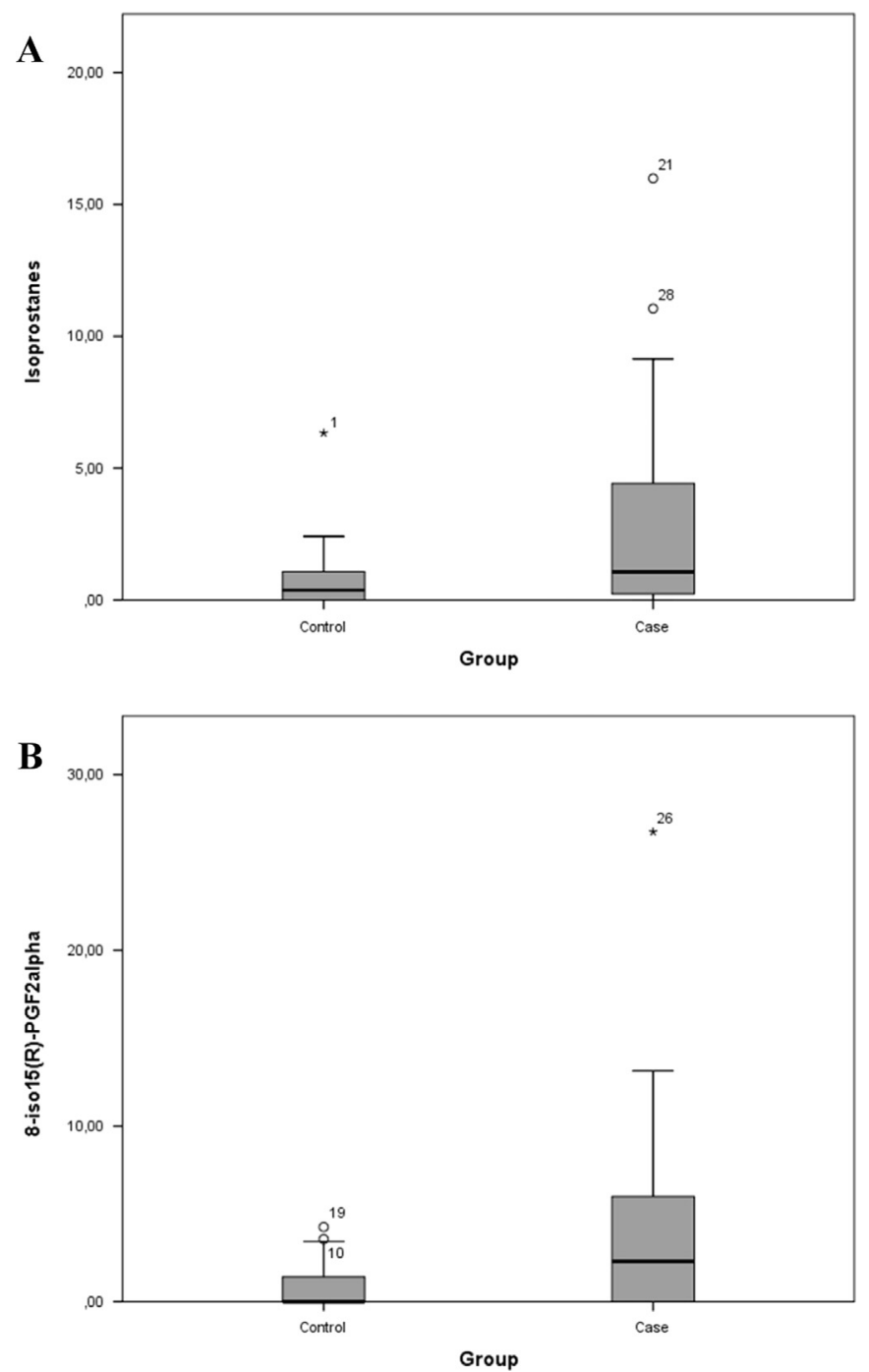

Fig. 1. Box plot graphs representing the concentration in cord blood serum samples for: (a) Isoprostanes, (b) 8-iso-15(R)-PGF2 $\alpha$ in control and case groups. Boxes represent the 1st and the 3rd quartiles, the black lines the median, and whiskers encompass from the 9 th to 91 st percentiles.

hypoxanthine as substrate generates a burst of oxygen free radicals, especially anion superoxide causing oxidative stress and damage to nearby molecules such as proteins, lipids and DNA [29]. The deleterious effects of oxygen free radicals upon the brain have been firmly established in clinical studies in the newborn period. Thus, conditions such as hypoxic ischemic encephalopathy have been associated with increased generation of free radicals and oxidative stress derived metabolites. Vasilyevich et al. measured the activity of glutathione peroxidase (GPX) in cerebrospinal fluid (CSF) of newborn infants with HIE [31]. GPX is an essential component of the glutathione redox enzyme system that reduces superoxide anion into hydrogen peroxide. An increased activity of GPX indirectly reveals the existence of a pro-oxidant status and oxidative stress. Remarkably, GPX activity in CSF correlated with clinical stage of HIE, CSF concentration of neuron specific enolase a marker of neuronal damage, and with subsequent neurological outcomes revealing that oxidative stress is a decisive agent in the development of HIE [31]. Kumar et al., determined malondialdehyde (MDA) and nitrate/nitrite levels in plasma of 43 term newborn babies who subsequently developed HIE [32]. MDA is a lipid peroxidation byproduct widely employed in assessing oxidative damage to lipids. Moreover, they assessed the integrity of 
A
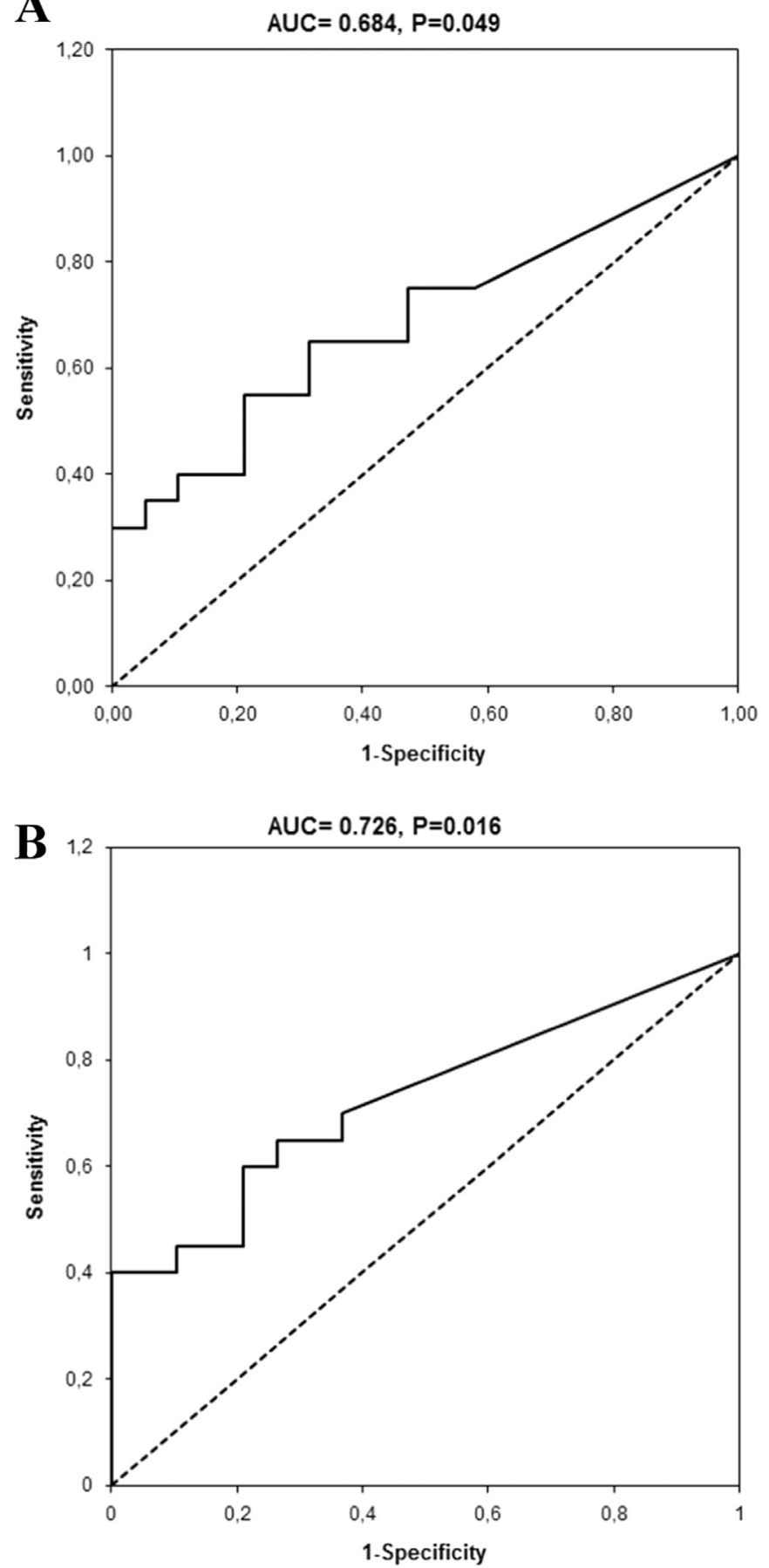

Fig. 2. Receiver operating characteristic (ROC) curves for: (a) Isoprostanes, (b) 8 -iso-15(R)-PGF2 $\alpha$.

the blood brain barrier measuring the cerebrospinal fluid albumin/ plasma albumin ratio. Their findings confirmed a significantly increased concentration of MDA, nitrate/nitrite levels, and CSF/ plasma albumin ratio in asphyxiated babies as compared to normal controls, concluding that both oxidative stress and increased permeability of the blood brain barrier played a relevant role in the pathogenesis of HIE [32]. Ogihara et al. [33], established a significant correlation between the levels of ortho-tyrosine and meta-tyrosine in CSF of babies evolving to HIE after an asphyctic episode and the presence of non-protein bound iron (NPBI). Circulating phenylalanine oxidation is a highly reliable marker of hydroxyl derived protein oxidation; moreover, in vivo production of hydroxyl radicals requires the presence of transition metals
Table 3

Diagnostic indices for individual biomarkers in the prediction of metabolic acidosis in asphyxiated newborn infants.

\begin{tabular}{lcc}
\hline & 8-iso-15(R)-PGF2 $\alpha$ & Isoprostanes \\
\hline AUC $(95 \% \mathrm{CI})$ & $0.726(0.566-0.887)$ & $0.684(0.516-0.853)$ \\
Sensitivity $(\%, 95 \% \mathrm{CI})$ & $40(19-64)$ & $55(34-74)$ \\
Specificity (\%, 95\% CI) & $100(82-100)$ & $79(57-91)$ \\
PPV $(\%, 95 \% \mathrm{CI})$ & $100(63-100)$ & $62(45-92)$ \\
NPV $(\%, 95 \% \mathrm{CI})$ & $61(42-78)$ & $2.6(1.0-6.8)$ \\
LR+ (95\% CI) & $15.6(0.96-253)$ & $0.57(0.33-0.98)$ \\
LR- $(95 \% \mathrm{CI})$ & $0.60(0.42-0.86)$ & $4.58(1.12-18.80)$ \\
DOR $(95 \% \mathrm{CI})$ & $25(1.3-483)$ & \\
\hline
\end{tabular}

AUC, area under the curve; PPV, positive predictive value; NPV, negative predictive value; $\mathrm{LR}+$, positive likelihood ratio; LR-, negative likelihood ratio; $\mathrm{CI}$, confidence interval; DOR, diagnostic odds ratio.

(iron, copper, manganese, etc.) as catalyzing agents and a biologic reducing agent such as anion superoxide or ascorbic acid. Newborn infants with severe HIE had significantly increased nonprotein bound iron, ortho-and-meta-tyrosine, and ascorbic acid levels in CSF as compared to normal controls. Moreover, metabolite levels correlated with the severity of HIE [33]. Shouman et al. [34] also found increased NPBI and MDA CSF and serum levels in babies with asphyxia evolving to HIE, and established a correlation between clinical severity and metabolite concentration.

Brain tissue is especially rich in lipid components in their cell membranes. Therefore oxidative derivatives of polyunsaturated fatty acids, such as, arachidonic and docosahexanoic acids, are considered extremely susceptible and therefore, highly specific of oxidative aggression to the central nervous system. Moreover, isoprostanes are highly stable compounds as compared to aldehydes (MDA) or peroxyl radicals, and can be measured in all biologic fluids [15]. Significant elevation of isoprostanes has been detected in neurodegenerative diseases [35], and HIE [15]. Our group has developed and validated highly reliable methods based on ultra-performance liquid chromatography coupled to tandem mass spectrometry to determine prostaglandin-like and derivatives in plasma, serum, and urine of newborn infants $[19,30]$. We wanted to establish a correlation between severe metabolic acidosis and increased prostanoidderivatives as a first step to identify possible early and reliable markers of brain damage. In our study, patients from case group had a significant metabolic acidosis and clinical signs of asphyxia as compared with those from control group (see Table 1). When comparing cord serum samples, we found that 8-iso-15(R)-PGF2 $\alpha$ and total isoprostanes had a highly significant correlation with severe postnatal metabolic acidosis. Actually, increased brain levels of 8-epi-PGF2a were found in asphyctic newborn rats $2 \mathrm{~h}$ after global perinatal asphyxia, and this biomarker could be predictive of delayed behavioral disturbances [22]. These biomarkers have several advantages, such as establishing a quantitative result and diagnosis to clinically undefined samples, and being potential long-term neurological damage biomarkers [14,22]. This fact can be explained by the molecular damage occurred under hypoxic episode depending on its intensity, duration, previous metabolic state, gestational age, and the degree of development of the antioxidant system, altogether these factors can induce a different response to a hypoxic episode. Thus, we considered that the different biomarker levels found in cord serum samples could reflect this multifactor-dependent oxidative stress damage. Interestingly, while standard clinical and biochemical variables return to normal non-hypoxic values after $4 \mathrm{~h}$, peroxidation biomarkers still remain elevated at this stage [16]. Therefore, these biomarkers could be determined during the first hours after birth and in peripheral blood samples helping clinicians to take more accurately therapeutic decisions.

There are limitations in the present study. The first one is the small sample size employed to study potential biomarkers. However, 
the incidence of newborn infants with severe metabolic acidosis is not so frequent, and we only chose those located in the extreme of severity. In addition, being a pilot study we just wanted to identify potential biomarkers. A second limitation is the lack of information relative to neurocognitive outcomes. Babies included in the study are being followed and a correlation between biomarkers and neurocognitive follow up will be established.

\section{Conclusions}

This is the first study that looks for biomarkers that correlate with the most widely employed clinical variables to diagnose metabolic acidosis in asphyxiated term newborn infants. The metabolite 8-iso-15(R)-PGF2 $\alpha$ and total isoprostanes showed suitable diagnostic indices. From these compounds a multivariate model, which takes into account all the possible variables related to the different biomarkers levels and oxidative stress damage, was performed, improving the correlation with the clinical variables and obtaining very good diagnostic indices. Further research will be carried out in an adequately powered prospective study to evaluate simultaneously these new biomarkers as prognostic test of adverse neurological results triggered by perinatal asphyxia.

\section{Conflicts of interest}

The authors disclose not conflicts of interest.

\section{Acknowledgements}

MV acknowledges PI14/0443, RD12/0026/0012 from the Health Research Institute Carlos III and RETICS funded by the PN 20182011 (Spain), ISCIII- Sub-Directorate General for Research Assessment and Promotion, and the European Regional Development Fund (FEDER), reference RD12/0026. CC-P acknowledges postdoctoral Carmen y Severo Ochoa research grant from the City Government of Valencia (Spain). The study was also supported by grants from Finska Läkaresällskapet, The Foundation for Pediatric Research in Finland, Päivikki and Sakari Sohlberg Foundation, and by Government Subsidy for Clinical Research.

\section{References}

[1] M.V. Johnston, W.H. Trescher, A. Ishida, W. Nakajima, Neurobiology of hypoxic-ischemic injury in the developing brain, Pediatr. Res. 49 (2001) 735-741.

[2] N. Kathri, H.Y. Man, Synaptic activity and bioenergy homeostasis: implications in brain trauma and neurodegenerative diseases, Front. Neurol. 4 (2013) 199.

[3] G. Wassink, E.R. Gunn, P.P. Drury, L. Bennet, A.J. Gunn, The mechanisms and treatment of asphyxial encephalopathy, Front. Neurosci. 8 (2014) 40.

[4] M. Douglas-Escobar, M.D. Weiss, Hypoxic-ischemic encephalopathy: a review for the clinician, JAMA Pediatr. 169 (2015) 397-403.

[5] O.D. Saugstad, Is lactate a reliable indicator of tissue hypoxia in the neonatal period? Acta Paediatr. 91 (2002) 16-19.

[6] G.L. Malin, R.K. Morris, K.S. Khan, Strength of association between umbilical cord $\mathrm{pH}$ and perinatal and long term outcomes systematic review and metaanalysis, Br. Med. J. 340 (2010), c1471.

[7] M.C. Lai, S.N. Yang, Perinatal hypoxic-ischemic encephalopathy, J. Biomed. Biotechnol. 2011 (2011) 1-6.

[8] C. Skappak, S. Regush, P.Y. Cheung, D.J. Adamko, Identifying hypoxia in a newborn piglet model using urinary NMR metabolomic profiling, PloS One 8 (2013) e65035.

[9] A. Leviton, O. Dammann, Brain damage markers in children. Neurobiological and clinical aspects, Acta Paediatr. 91 (2002) 9-13.

[10] L. Bennet, L. Booth, A.J. Gunn, Potential biomarkers for hypoxic-ischemic encephalopathy, Semin. Fetal Neonatal Med. 15 (2010) 253-260.

[11] M.V. Douglas-Escobar, S.C. Heaton, J. Bennett, L.J. Young, O. Glushakova, X. Xu, D.Y. Barbeau, C. Rossignol, C. Miller, A.M. Old Crow, R.L. Hayes, M.D. Weiss, UCH-L1 and GFAP serum levels in neonates with hypoxic-ischemic encephalopathy: a single center pilot study, Front. Neurol. 5 (2014) 273.

[12] M. Zaigham, F. Lundberg, R. Hayes, J. Undén, P. Olofsson, Umbilical cord blood concentrations of ubiquitin carboxy-terminal hydrolase L1 (UCH-L1) and glial fibrillary acidic protein (GFAP) in neonates developing hypoxic-ischemic encephalopathy, J. Matern. Fetal Neonatal Med. 10 (2015) 1-7.

[13] A.M. Looney, C. Ahearne, G.B. Boylan, D.M. Murray, Glial fibrillar acidic protein is not an early marker of injury in perinatal asphyxia and hypoxic-ischemic encephalopathy, Front. Neurol. 6 (2015) 264.

[14] M.S. Rogers, C.C. Wang, T.L. Lau, X. Xiao, X.G. Zhou, T.F. Fok, K.O. Chu, C.P. Pang, Relationship between isoprostane concentrations, metabolic acidosis, and morbid neonatal outcome, Clin. Chem. 51 (2005) 1271-1274.

[15] M.L. Tataranno, S. Perrone, G. Buonocore, Plasma biomarkers of oxidative stress in neonatal brain injury, Clin. Perinatol. 42 (2015) 529-539.

[16] C. Fattuoni, F. Palmas, A. Noto, V. Fanos, L. Barberini, Perinatal asphyxia: a review from a metabolomics perspective, Molecules 20 (2015) 7000-7016.

[17] J. Meyburg, A. Schulze, D. Kohlmueller, O. Linderkamp, E. Mayatepek, Postnatal changes in neonatal acylcarnitine profile, Pediatr. Res. 49 (2001) 125-129.

[18] G. Tonni, S. Leoncini, C. Signorini, L. Ciccoli, C. De Felice, Pathology of perinatal brain damage: background and oxidative stress markers, Arch. Gynecol. Obstet. 290 (2014) 13-20.

[19] J. Kuligowski, J. Escobar, G. Quintás, I. Lliso, I. Torres-Cuevas, A. Nuñez, E. Cubells, D. Rook, J.B. van Goudoever, M. Vento, Analysis of lipid peroxidation biomarkers in extremely low gestational age neonate urines by UPLC-MS/MS, Anal. Bioanal. Chem. 406 (2014) 4345-4356.

[20] G.L. Milne, Q. Dai, L.J. Roberts, The isoprostanes-25 years later, Biochim. Biophys. Acta 1851 (2015) 433-445.

[21] H. Sakamoto, T.B. Corcoran, J.G. Laffey, G.D. Shorten, Isoprostanes markers of ischaemia-reperfusion injury, Eur. J. Anaesthesiol. 19 (2002) 550-559.

[22] G. Calamandrei, A.P. Venerosi, A. Valanzano, M.A. de Berardinis, A. Greco, M. Puopolo, L. Minguetti, Increased brain levels of F2-isoprostane are an early marker of behavioral sequels in a rat model of global perinatal asphyxia, Pediatr. Res. 55 (2004) 85-92.

[23] C. Kerkhofs, C. De Bruyn, T. Mesens, C. Theyskens, M. Vanhoestenberghe, E. Bruneel, C. Van Holsbeke, A. Bonnaerens, W. Gyselaers, Identification of peripartum near-miss for perinatal audit, Facts Views Vis. Obgyn 6 (2014) 177-183.

[24] J.A. Low, C. Panagiotopoulos, E.J. Derrick, Newborn complications after intrapartum asphyxia with metabolic acidosis in the term fetus, Am. J. Obstet. Gynecol. 170 (1994) 1081-1087.

[25] L. Knutzen, E. Svirko, L. Impey, The significance of base deficit in acidemic term neonates, Am. J. Obstet. Gynecol. 213 (2015) e1-e7.

[26] M.G. Tuuli, M.J. Stout, A. Shanks, A.O. Adibo, G.A. Macones, A.G. Cahill, Umbilical cord arterial lactate compared with $\mathrm{pH}$ for predicting neonatal morbidity at term, Obstet. Gynecol. 124 (2014) 756-761.

[27] P. Yeh, K. Emary, L. Impey, The relationship between umbilical cord arterial pH and serious adverse neonatal outcome: analysis of 51,519 consecutive validated samples, BJOG 119 (2012) 824-831.

[28] A.G. Cahill, Umbilical artery pH and base deficit in obstetrics, Am. J. Obstet. Gynecol. 213 (2015) 257-258.

[29] E. Maltepe, O.D. Saugstad, Oxygen in health and disease: regulation of oxygen homeostasis-clinical implications, Pediatr. Res. 65 (2009) 261-268.

[30] C. Chafer-Pericas, L. Rahkonen, A. Sánchez-Illana, J. Kuligowski, I. TorresCuevas, M. Cernada, E. Cubells, A. Nuñez-Ramiro, S. Andersson, M. Vento, J. Escobar, Ultrahigh performance liquid chromatography coupled to tandem mass spectrometry determination of lipid peroxidation biomarkers in newborn serum samples, Anal. Chim. Acta 886 (2015) 214-220.

[31] B. Vasiljevic, S. Maglajlic-Djukic, M. Gojnic, S. Stankovic, The role of oxidative stress in perinatal hypoxic-ischemic brain injury, Srp. Arh. Celok. Lek. 140 (2014) 35-41.

[32] A. Kumar, R. Mittal, H.D. Khanna, S. Basu, Free radical injury and blood brain barrier permeability in hypoxic-ischemic encephalopathy, Pediatrics 122 (2008) e722-e727.

[33] T. Ogihara, K. Hirano, H. Ogihara, K. Misaki, M. Hiroi, T. Morinobu, H.S. Kim, S. Ogawa, R. Ban, M. Hasegawa, H. Tamai, Hydroxyl radical generation in cerebrospinal fluid of newborn infants with hypoxic-ischemic encephalopathy, Pediatr. Res. 53 (2003) 594-599.

[34] B.O. Shouman, A. Mesbah, H. Aly, Iron metabolism and lipid peroxidation products in infants with hypoxic ischemic encephalopathy, J. Perinatol. 28 (2008) 487-491.

[35] E. Miller, A. Morel, L. Saso, J. Saluk, Isoprostanes and neuroprostanes as biomarkers of oxidative stress in neurodegenerative diseases, Oxid. Med. Cell. Longev. 2014 (2014) 572491. 